\title{
Methodological variations in the production of CORINE Land Cover and consequences for long-term land cover change studies. The case of Spain.
}

Running head: Long-term impact of CORINE LC methodological variations

\section{Authors and affiliations:}

Jesús Martínez-Fernández ${ }^{1}$, Paloma Ruiz-Benito ${ }^{2,3}$, Andreu Bonet ${ }^{4}$, Cristina Gómez ${ }^{1,5 *}$

${ }^{1}$ INIA, Forest Research Centre, Ctra. de La Coruña km 7.5, 28040 Madrid, Spain.

${ }^{2}$ Departamento de Biología y Geología, Física y Química Inorgánica, Escuela Superior de Ciencias Experimentales y Tecnología, Universidad Rey Juan Carlos, C/ Tulipán s/n, 28933, Móstoles, Spain

${ }^{3}$ Grupo de Ecología y Restauración Forestal, Departamento de Ciencias de la Vida, Universidad de Alcalá, Edificio de Ciencias, Campus Universitario, 28805 Alcalá de Henares Madrid, Spain.

${ }^{4}$ Department of Ecology, University of Alicante, Ctra. de San Vicente del Raspeig s/n 03690 Alicante, Spain.

${ }^{5}$ Department of Geography and Environment, School of Geoscience, University of Aberdeen, Aberdeen AB24 3UE, UK.

*Corresponding author: c.gomez@abdn.ac.uk

\section{ORCIDS:}

Jesús Martínez-Fernández: 0000-0002-0686-6206

Paloma Ruiz-Benito: 0000-0002-2781-5879

Andreu Bonet: 0000-0002-4210-9966

Cristina Gómez: 0000-0002-2756-0863

Submitted to: International Journal of Remote Sensing

Submitted on: $6^{\text {th }}$ November 2018

Revision submitted on: $6^{\text {th }}$ March 2019

Accepted: $24^{\text {th }}$ March 2019

\section{Pre-print of published version}

Jesús Martínez-Fernández, Paloma Ruiz-Benito, Andreu Bonet \& Cristina Gómez (2019): Methodological variations in the production of CORINE Land Cover and consequences for long-term land cover change studies. The case of Spain., International Journal of Remote Sensing.

\section{DOI: https://doi.org/10.1080/01431161.2019.1624864}

\section{Disclaimer:}

The PDF document is a copy of the final version of this manuscript that was subsequently accepted by the journal for publication. The paper has been through peer review, but has not been subject to any additional copy-editing or journal specific formatting (so will look different from the final version of record, which may be accessed following the DOI above depending on your access situation). 


\title{
Methodological variations in the production of CORINE Land Cover and consequences for long-term land cover change studies. The case of Spain.
}

\begin{abstract}
Land cover information at national or regional scale is essential for science, monitoring, reporting, and policy making. CORINE Land Cover (CLC) is the most consistent land cover map for the entire European territory, with four repetitions during the period 1985-2012. The long-term consistency of CLC maps is the most appreciated strength and it should be guaranteed. Beyond some common general guidelines, the mapping approaches used in individual countries differ and change over time, leading to inconsistencies that should be known and reported. Through a series of metrics over the Spanish CLC most recent layers (CLC2006 and CLC2012) and comparisons with eight other countries CLC statistics we demonstrate that the methodological changes recently implemented have introduced some discrepancies with previous CLC versions. The most affected classes in Spain were transitional woodland-shrub, complex cultivation patterns, artificial, grasslands, and forests. Users should be aware of the important implications these discrepancies may have in land use and land cover change studies, trend analysis, and reports.
\end{abstract}

Keywords: CORINE Land Cover, land cover change, Spain, cartographic validation

\section{Introduction}

Land cover (LC) maps are essential baseline data for environmental research (Turner et al., 2007) and policy support (Maes et al., 2012). Series of LC maps representing the Earth surface condition at different times enable the assessment of terrestrial dynamics, and are necessary to derive environmental indicators of change, and for planning and management of natural resources. Maps in a LC series must be comparable and they should provide measurements of accuracy, homogeneity, uncertainties and limitations (Büttner 2014). Transparency in the mapping methods is necessary to assess the compatibility of a series of LC maps (Weiers et al., 2002; Gallego et al., 2000).

CORINE Land Cover (CLC) is a wall-to-wall LC map at 1:100000 scale for the entire territory of the European Union (EU) Member States and associate countries (currently 39 countries and approximately 5.8 million squared kilometres). CLC is the most complete and consistent cartographic source of knowledge about the changes that occurred in the European landscape during the reference period 1985-2012 (Feranec et al., 2016a). CLC has standard specifications (e.g. minimum mapping unit-MMU of 25.0 ha, corridors of $100 \mathrm{~m}$ minimum width) and a homogeneous land cover scheme-comprising 44 classes in a three-level hierarchy - and nomenclature through Europe (Heymann et al., 1994), which has remained unchanged since the implementation of the first CORINE for the 1990 epoch. CLC has been updated three times for years 2000 (CLC2000), 2006 (CLC2006) and 2012 (CLC2012) (Bossard et al., 2000; EEA 2007; Büttner 2014) and a fifth version (CLC2018) is currently available and in its final validation stages (EEA, 2018). The base images for developing CLC span a number of years, different for each country and CLC version. Overall, CORINE Land Cover 1990 (CLC1990) layer results from interpretation of images acquired from 1985 to 1998 (Soukup et al., 2016). In the case of Spain CLC1990 employs images acquired between 1984 and 1990, although most were acquired in 1987 (OSE, 2006). Later CLC versions have 
employed shorter time interval materials. Thanks to its periodicity and consistency over time CLC is especially appropriate for reports and analysis at national or regional scale (MartínezFernández et al., 2015). The temporal and spatial consistency of CLC map series is its most appreciated characteristic, and it should be ensured and maintained (García-Álvarez and Camacho, 2017).

CLC is produced at national level, with Earth Observation satellite images as the main source of data. Beyond some general guidelines established by the European Environmental Agency (EAA), each country implements the methodology that best adjusts to its particular circumstances (e.g. data availability, processing resources) for production of the CLC maps. Differences in mapping approaches may result in spatial and/or temporal disparities and inconsistences that should be known and considered in comparative reports. Therefore, technical studies reporting methodological limitations and the consequences for analysis and interpretation of results become necessary, especially when there are changes in mapping methods over time (Hazeu et al., 2016).

\subsection{Overall Evolution in CLC Production Methodology in European Countries}

CLC1990 was derived by visual Photo-Interpretation (PI) of satellite images hard copies using plastic overlays. This technology, employed by the 27 countries participating in the CLC1990 project, was discarded and replaced by Computer-Assisted Photo-Interpretation (CAPI) during the CLC2000 project. Semiautomatic generalization (SAG) methods were applied for the first time in the CLC2000 by Switzerland, Finland, Norway, Sweden, Iceland, and the United Kingdom (UK) (Hazeu et al., 2016), all of them participating in the CLC project for the first time (i.e., they do not have CLC1990). SAG methods imply the generalization of more detailed national LC maps and Geographic Information Systems (GIS) integration of elementary in situ data (Feranec et al., 2016b). Since CLC2000 a change layer (CLCchange) is produced between CLC versions, with MMU of 5.0 ha. In CLC2006, 33 out of 38 participating countries applied CAPI methods (Feranec et al., 2016b). For the CLC2012 update a few countries - Germany, Ireland, Austria, and Spain - have moved from standard CAPI toward SAG approaches of CLC production (Hovenbitzer et al 2014; GarcíaÁlvarez and Camacho Olmedo 2017). However, the UK reverted from SAG to CAPI from 2006 to 2012 because a national dataset necessary to perform the generalization to CLC2012 was not available (Cole et al., 2015). Despite having more detailed land use and land cover (LULC) datasets available, the Netherlands has preferred to maintain the independence between national LULC data generation and the CLC project (Hazeu et al., 2016). With changes in the production methodology countries like Germany, Ireland, Austria, Spain, and the UK may lose the consistency of the CLC time series, and should search for methods to compare the different CLC layers, assessing the impact of methodological changes.

\subsection{Changes in CLC Production Methodology in Spain}

In Spain CLC inventories from 1990 to 2006 were generated by visual PI or CAPI of satellite images and orthophotos, supplemented with existing national thematic data (Hazeu et al, 2016; OSE, 2006). In contrast, CLC2012 has been produced from the generalization of SIOSE2011 (Sistema de Información de Ocupación del Suelo en España), a more detailed LULC map produced since 2005 at 1:25000 scale and with MMU of 0.5-2.0 ha. The updating 
process of any CLC map requires revising the immediately preceding product and deriving a revised version. Therefore, when mapping CLC2012, CLC2006revised was produced with identical methodology to CLC2012, by a generalization of SIOSE2005. CLC2012 and CLC2006revised are methodologically identical and therefore properly comparable. CLC2000 and CLC2006initial are also directly comparable, because both were generated by CAPI. In contrast, long-term studies comparing early layers and recent layers may find discrepancies related to methodological issues rather than real LC changes. For instance, some disagreement between CLC2012 and CLC2006initial is caused by a different precision in the delimitation of polygon perimeters, because CLC2012 sketches them from the SIOSE 1:25000 scale, producing a high degree of complexity and detail not expected at 1:100000 scale. CLC2012 has more fragmentation and number of patches, and more irregular polygons, as well as a large number of narrow corridors ( $<100 \mathrm{~m}$ width) than previous CLC versions (García-Álvarez and Camacho, 2017). Therefore, for the 2006 epoch it is possible to compare two CLC maps derived applying different methods: (1) CLC2006initial by traditional CAPI, and (2) CLC2006revised by SAG of SIOSE2005. When comparing these two versions the reference closer to reality is CLC2006revised because SIOSE is more precise and has finer scale.

Our goal is to contribute to the search of robust and transparent methods to compare CLC maps over time, enabling the use of comparative products with awareness of their strengths and limitations. In this work we provide basic reference statistics of LC and change at the national level for Spain, facilitating the establishment of trends in LC classes over the entire CLC life period, and highlighting LC discrepancies before and after 2006 due to the change produced in methodological production. Since other European countries have also changed their CLC production methodology, we contextualize the problem comparing a range of metrics and methodological approaches in nine European countries.

\section{Data and Methods}

\subsection{Comparison of the Spanish CLC Layers before and after 2006}

Both CLC2006 map versions, CLC2006initial (produced by CAPI) and CLC2006revised (produced by SAG) for the entire national territory of Spain (50.6 million ha) were compared using GIS software with tools for query, selection, intersection, and cross-tabulation. CLC GIS layers were downloaded from the most updated sites. CLC2006initial for Spain was obtained from the Spanish National Geographic Information Centre (CNIG) Download Service (CNIG, 2013) and for other European countries from the EEA webpage, corresponding to version 17 (EEA, 2014). CLC2006revised, CLC2012, and CLC2000 were downloaded from the Copernicus Land Monitoring Service, corresponding with version 18.5.1 (EEA, 2016). CLC2006revised is not available in the Spanish CNIG.

For characterization of LC in both CLC2006 versions, some basic metrics of all classes and hierarchical levels were calculated: 1) number of polygons $\left.\left(N_{\text {pol }}\right), 2\right)$ absolute area, 3) relative area with respect to the total area of Spain, and 4) relative Net Change (NC) as proposed by Pontius et al. (2004). NC represents the variation of a LC class relative to its area at the start of the period. NC is a useful metric to identify the degree of changeregardless of the absolute value-enabling appreciation of changes in rare classes.

Interpretation of these basic metrics enables assessing the agreement between 
CLC2006initial and CLC2006revised and shows which classes have been overestimated or underestimated in CLC2006initial with respect to the reference, in this case CLC2006revised.

The accuracy of the change identification was evaluated via confusion matrix, and conflicts between individual classes were reported interpreting the omission and commission errors as typically done in cartographic validation (Chuvieco 2010). In this study, CLC2006revised is considered true (i.e., the reference layer or "ground truth") for assessment of commission and omission error in CLC2006initial. Focusing on a particular LC class, a pixel misclassified incurs in omission or "exclusion" error when CLC2006initial does not identify the real CLC2006revised class, whereas commission or "inclusion" errors include pixels that CLC2006initial identifies as the target LC but are a different class in CLC2006revised. The omission and commission errors express two approaches to the same problem. Omission refers to an underestimation of a particular class while commission error is caused by an overestimation in CLC2006initial classes. Omission and commission errors were related to the target class underestimation and overestimation respectively.

\subsection{Differences in European Countries}

To contextualize the Spanish case we looked into the impact of the changes in CLC production methodology in other countries. All available technical information for the last four CLC versions or updates in Germany, Austria, Ireland, the UK, Finland, France, Portugal, and the Netherlands was compiled and summarized. Exploring CLC2000, CLC2006initial, CLC2006revised, and CLC2012 layers, changes were assessed with a dual approach: 1) comparing the differences between CLC level 1 class proportions percentage respect the country area) for each of the nine countries and 2) through visual analysis with a map composition at scale 1:150000 for six countries. To facilitate the statistical comparison we employed also the Sum Difference Value (SDV), an indicator representing the summation of absolute differences between each class percentage in CLC2006revised (CLC2006 ${ }_{\mathrm{r}}$ ) and CLC2006initial (CLC2006 $)$.

$$
\mathrm{SDV}=\sum\left|\mathrm{CLC} 2006_{i}(\%)-\mathrm{CLC}_{2} 006_{r}(\%)\right|
$$

SDV values provide an estimation of the overall disagreement between pairs of maps for an entire country, and allows straight forward comparison of differences between countries. Statistical data of level 1 LC classes (artificial, agricultural, forests, wetlands, and water bodies) at the country level are available for CLC2006initial (EEA, 2011) and for CLC06revised (EEA, 2017).

\section{Results}

\subsection{Comparison of the Spanish CLC Layers before and after 2006}

Land cover statistics comparing CLC2006initial and CLC2006revised class areas at level 2 (15 classes) and level 3 (44 classes) are detailed in Tables 1 and 2. An underestimation of artificial and forest areas by CLC2006initial is observed, whilst agricultural, wetlands, and water bodies were overestimated (Table 1). The agricultural overestimation was overall produced in heterogeneous areas (code 24) (6.4\% higher in the initial version), particularly 
in complex cultivation patterns (code 242, Table 2) (4.0\% higher) and agriculture with areas of natural vegetation (code 243, Table 2). As an exception, the area of agroforestry systems (code 244) was very similar in both 2006 versions.

Among forest ecosystems CLC2006initial overestimated herbaceous and scrub classes (code 32, Table 1) irregularly among subclasses (Table 2), since transitional woodland-scrub (324) was highly overestimated, whereas natural grasslands (321) and moors and heathlands (322) were underestimated (Table 2). Forests in general, and particularly broadleaved forests (311) were considerably underestimated (Tables 1 and 2).

Wetlands and water bodies can be considered rare classes, constituting a small proportion of the national territory (less than $0.1 \%$ ), and were overestimated in CLC2006inital. This overestimation is at least in part due to the less precise PI techniques. As an exception, peat bogs (412), salt marshes (421) and water courses (511) were underestimated in the CLC2006initial version (Table 2). SIOSE2005 (base for developing CLC2006revised) maps lineal elements over $15 \mathrm{~m}$ width, whereas CLC2006initial had the $100 \mathrm{~m}$ wide restriction to map linear elements.

Artificial areas occupy less than $2.3 \%$ of the national territory and can also be considered rare classes. However, the underestimation of artificial classes by CLC2006initial is relevant, as revealed by NC values (Tables 1 and 2). CLC2006revised has improved the identification of these areas with an increment, especially those represented by small polygons and/or with complicated perimeters (e.g. discontinuous urban fabric (112), green urban (141), sport and leisure facilities (142), industrial or commercial units (121), and road and rail networks (122)). These classes have probably gained area to other previously overestimated classes like continuous urban fabric (111), port areas (123), airports (124), and mineral extraction sites (131)

Figure 1 shows the Spanish level 1 LC trends from CLC1990 to CLC2012. Dashed vertical lines show the trends' breaks caused by methodological changes starting with CLC2006revised and onwards. We propose using this kind of graph in long-term LC studies, as they are readily understood and provide visual information to discern between real changes and changes due to technical issues. Some LC classes are more affected by the change in methodology than others. To identify the discrepancies that occurred between the initial and revised CLC2006 versions, an extract of the confusion matrix is shown in Table 3, with commission $(C)$ and omission $(O)$ errors for the seven most affected LC classes according to national values shown in Table 2. For detailed interpretation see the first column as an example: The commission error of complex cultivation patterns was $19.3 \%$, that is, almost one fifth of complex cultivation patterns in the initial version were in reality non-irrigated arable land in the revised version, whereas omission error was $8.7 \%$, that is, non-irrigated arable land in the initial LC and complex cultivation patterns in the revised version. In this case, according to Table 2, this class has been overestimated in CLC2006initial, and therefore commission error is more important than omission error. However, in the case of underestimated classes as broad-leaved forest, the omission errors are more important. Three LC classes are particularly overestimated in CLC2006initial: 1) complex cultivation patterns (242) — confused particularly with non-irrigated arable land (211) and permanent crops $(22)-, 2)$ agriculture with areas of natural vegetation (243) — confused with non-irrigated arable land (211), sclerophyllous vegetation (323), forests (31), and natural grasslands (321), as well as complex cultivation (242)—, and 3) transitional woodland-shrub (324)—confused with forests (31) and the rest of scrubs and herbaceous vegetation (32). On the other hand, there are four classes particularly underestimated in the CLC2006initial version: 1) 
broadleaved forest (311) and 2) conifers (312)-confused with mixed forest (313), sclerophyllous vegetation (323), and transitional woodland-shrub (324) - , 3) natural grasslands (321) — confused with the rest of scrub classes $(322,323$, and 324) and nonirrigated arable land (211) — , and 4) moors and heathlands (322) — confused with transitional woodland-shrub (324), natural grasslands (321), sclerophyllous vegetation (323) and broadleaved forests (311) — (see Table 3$)$.

Table 1. CLC level 1 and level 2 statistics for CLC2006initial and CLC2006revised. $N_{\text {pol }}$ is the number of polygons in each class. Percentages are calculated with respect to the total area of Spain. Difference (\%) is Initial (\%) - Revised (\%). Difference values over $+/-1.00 \%$ are marked in bold. Classes underestimated in CLC2006initial with respect to CLC2006revised have negative differences. NC (\%) is defined as (Difference $(\%) /$ Initial (\%)) $\times 100$. SDV (see Equation 1) is indicated with * in the row Total.

\begin{tabular}{|c|c|c|c|c|c|c|c|c|}
\hline \multirow{2}{*}{$\begin{array}{l}\text { Land cover class } \\
\text { Code and Name }\end{array}$} & \multicolumn{3}{|c|}{ CLC2006initial } & \multicolumn{3}{|c|}{ CLC2006revised } & \multirow{2}{*}{$\begin{array}{c}\text { Difference } \\
(\%)\end{array}$} & \multirow{2}{*}{$\begin{array}{l}\mathrm{NC} \\
(\%)\end{array}$} \\
\hline & $N_{\mathrm{pol}}$ & Area (ha) & $\begin{array}{c}\text { Initial } \\
(\%)\end{array}$ & $N_{\mathrm{pol}}$ & Area (ha) & $\begin{array}{c}\text { Revised } \\
(\%)\end{array}$ & & \\
\hline 1 ARTIFICIAL SURFACES & 11,304 & $1,017,368$ & 2.01 & 15,857 & $1,174.600$ & 2.32 & -0.31 & -15.5 \\
\hline 11 Urban fabric & 6,467 & 632,451 & 1.25 & 8,626 & 681,613 & 1.35 & -0.10 & -7.8 \\
\hline 12 Industrial, commercial and transport units & 2,149 & 197,373 & 0.39 & 3,587 & 264,631 & 0.52 & -0.13 & -34.1 \\
\hline 13 Mine, dump and construction sites & 2,212 & 152,741 & 0.30 & 2,829 & 173,370 & 0.34 & -0.04 & -13.5 \\
\hline 14 Artificial, non-agricultural vegetated areas & 476 & 34,803 & 0.07 & 815 & 54,986 & 0.11 & -0.04 & -58.0 \\
\hline 2 AGRICULTURAL AREAS & 64,097 & $25,364,366$ & 50.05 & 91,672 & $23,833,135$ & 47.04 & 3.00 & 6.0 \\
\hline 21 Arable land & 19,929 & $12,102,081$ & 23.88 & 29,399 & $12,684,457$ & 25.04 & -1.16 & -4.8 \\
\hline 22 Permanent crops & 10,916 & $3,594,360$ & 7.09 & 19,314 & $4,485,305$ & 8.85 & -1.76 & -24.8 \\
\hline 23 Pastures & 3,110 & 648,939 & 1.28 & 6,623 & 891,522 & 1.76 & -0.48 & -37.4 \\
\hline 24 Heterogeneous agricultural areas & 30,142 & $9,018,986$ & 17.80 & 36,336 & $5,771,851$ & 11.39 & 6.40 & 36.0 \\
\hline 3 FOREST and SEMI NATURAL AREAS & 94,318 & $23,852,293$ & 47.08 & 125,371 & $25,242,978$ & 49.82 & -2.77 & -5.8 \\
\hline 31 Forests & 32,445 & $9,139,235$ & 18.04 & 47,743 & $11,201,351$ & 22.11 & -4.08 & -22.6 \\
\hline 32 Scrub and/or herbaceous vegetation associations & 56,828 & $13,472,938$ & 26.59 & 70,542 & $12,909,566$ & 25.48 & 1.10 & 4.2 \\
\hline 33 Open spaces with little or no vegetation & 5,045 & $1,240,120$ & 2.45 & 7,086 & $1,132,061$ & 2.23 & 0.21 & 8.7 \\
\hline 4 WETLANDS & 428 & 111,083 & 0.22 & 1,538 & 99,369 & 0.20 & 0.02 & 10.5 \\
\hline 41 Inland wetlands & 165 & 54,960 & 0.11 & 127 & 44,516 & 0.09 & 0.02 & 19.0 \\
\hline 42 Maritime wetlands & 263 & 56,123 & 0.11 & 1,411 & 54,853 & 0.11 & 0.00 & 2.3 \\
\hline 5 WATER BODIES & 1,217 & 328,183 & 0.65 & 1,902 & 309,381 & 0,62 & 0.04 & 5.7 \\
\hline 51 Inland waters & 1,141 & 294,658 & 0.58 & 1,452 & 286,339 & 0.57 & 0.02 & 2.8 \\
\hline \multirow[t]{2}{*}{52 Marine waters } & 76 & 33,525 & 0.07 & 450 & 23,042 & 0.05 & 0.02 & 31.3 \\
\hline & 171,364 & $50,673,293$ & 100.00 & 236,340 & $50,659,463$ & 100.00 & $15.57 *$ & \\
\hline
\end{tabular}


Table 2. Land cover statistics by level 3 and comparison between CLC2006revised and CLC2006initial. $N_{\text {pol }}$ is the number of polygons in each class. Percentages are calculated with respect to the total area of Spain. Difference (\%) is Initial (\%) - Revised (\%). Difference values over $+/-1.00 \%$ are marked in bold. Classes underestimated in CLC2006initial with respect to CLC2006revised have negative differences. NC (\%) is defined as (Difference (\%) / Initial $(\%)) \times 100$. SDV (see Equation 1) is indicated with $*$ in the row Total.

\begin{tabular}{|c|c|c|c|c|c|c|c|c|}
\hline Land cover class & \multicolumn{3}{|c|}{ CLC2006initial } & \multicolumn{3}{|c|}{ CLC2006revised } & \multirow[b]{2}{*}{$\begin{array}{l}\text { Difference } \\
\%\end{array}$} & \multirow[b]{2}{*}{$\begin{array}{l}\mathrm{NC} \\
(\%)\end{array}$} \\
\hline Name & $N_{\mathrm{pol}}$ & Are & $\begin{array}{c}\text { Initial } \\
(\%)\end{array}$ & $N_{\text {pol }}$ & Area (ha) & $\begin{array}{c}\text { Revised } \\
(\%)\end{array}$ & & \\
\hline 111 Continuous urban fabric & 3,045 & 293,445 & 0.58 & 2,476 & 210,970 & 0.42 & 0.16 & 28.1 \\
\hline 112 Discontinuous urban fabric & 3,422 & 339,006 & 0.67 & 6,150 & 470,643 & 0.93 & -0.26 & -38.8 \\
\hline 121 Industrial or commercial units & 1,760 & 146,581 & 0.29 & 2,867 & 212,950 & 0.42 & -0.13 & -45.3 \\
\hline 122 Road and rail networks & 194 & 21,131 & 0.04 & 457 & 27,973 & 0.06 & -0.01 & -32.4 \\
\hline 123 Port areas & 126 & 10,633 & 0.02 & 192 & 6,884 & 0.01 & 0.01 & 35.3 \\
\hline 124 Airports & 69 & 19,028 & 0.04 & 71 & 16,824 & 0.03 & 0.00 & 11.6 \\
\hline 131 Mineral extraction sites & 1,071 & 75,565 & 0.15 & 1,194 & 76,414 & 0.15 & 0.00 & -1.1 \\
\hline 132 Dump sites & 87 & 5,914 & 0.01 & 255 & 12,144 & 0.02 & -0.01 & -105.4 \\
\hline 133 Construction sites & 1,054 & 71,263 & 0.14 & 1,380 & 84,811 & 0.17 & -0.03 & -19.0 \\
\hline 141 Green urban areas & 88 & 6,681 & 0.01 & 243 & 16,265 & 0.03 & -0.02 & -143.4 \\
\hline 142 Sport and leisure facilities & 388 & 28,122 & 0.06 & 572 & 38,721 & 0.08 & -0.02 & -37.7 \\
\hline 211 Non-irrigated arable land & 14,126 & $9,756,284$ & 19.25 & 21,054 & $10,078,857$ & 19.90 & -0.64 & -3.3 \\
\hline 212 Permanently irrigated land & 5,476 & $2,201,032$ & 4.34 & 8,049 & $2,469,043$ & 4.87 & -0.53 & -12.2 \\
\hline 213 Rice fields & 327 & 144,765 & 0.29 & 296 & 136,556 & 0.27 & 0.02 & 5.7 \\
\hline 221 Vineyards & 2,589 & 838,100 & 1.65 & 5,063 & $1,098,798$ & 2.17 & -0.52 & -31.1 \\
\hline $222 \begin{array}{l}\text { Fruit trees and berry } \\
\text { plantations }\end{array}$ & 3,244 & 891,091 & 1.76 & 6,695 & $1,134,787$ & 2.24 & -0.48 & -27.3 \\
\hline 223 Olive groves & 5,083 & $1,865,168$ & 3.68 & 7,556 & $2,251,721$ & 4.44 & -0.76 & -20.7 \\
\hline 231 Pastures & 3,110 & 648,939 & 1.28 & 6,623 & 891,522 & 1.76 & -0.48 & -37.4 \\
\hline 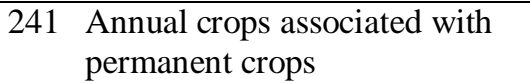 & 263 & 140 & 0.28 & 216 & 26 & 0.06 & 0 . & 80.1 \\
\hline 242 Complex cultivation patterns & 11,175 & $3,880,534$ & 7.66 & 13,909 & $1,835,867$ & 3.62 & 4.03 & 52.7 \\
\hline $\begin{array}{l}243 \text { Land principally occupied by } \\
\text { agriculture, with significant } \\
\text { areas of natural vegetation }\end{array}$ & 14,386 & $2,500,011$ & 4.93 & 16,107 & $1,429,742$ & 2.82 & 2.11 & 42.8 \\
\hline 244 Agro-forestry areas & 4,318 & $2,497,445$ & 4.93 & 6,104 & $2,478,216$ & 4.89 & 0.04 & 0.8 \\
\hline 311 Broad-leaved fores & 14,813 & $3,754,448$ & 7.41 & 22,377 & $5,125,372$ & 10.12 & -2.71 & -36.5 \\
\hline 312 Coniferous forest & 12,702 & $3,879,379$ & 7.66 & 16,837 & $4,665,454$ & 9.21 & -1.55 & -20.3 \\
\hline 313 Mixed forest & 4,930 & $1,505,407$ & 2.97 & 8,529 & $1,410,526$ & 2.78 & 0.19 & 6.3 \\
\hline 321 Natural grasslands & 10,703 & $2,644,987$ & 5.22 & 22,934 & $3,954,172$ & 7.81 & -2.59 & -49.5 \\
\hline 322 Moors and heathland & 3,376 & 932,742 & 1.84 & 7,751 & $1,980,299$ & 3.91 & -2.07 & -112.3 \\
\hline 323 Sclerophyllous vegetation & 18,596 & $5,231,940$ & 10.32 & 21,687 & $5,004,741$ & 9.88 & 0.45 & 4.3 \\
\hline 324 Transitional woodland-shrub & 24,153 & $4,663,269$ & 9.20 & 18,170 & $1,970,354$ & 3.89 & 5.31 & 57.7 \\
\hline 331 Beaches, dunes, sands & 342 & 48,023 & 0.09 & 339 & 37,651 & 0.07 & 0.02 & 21.6 \\
\hline 332 Bare rocks & 1,183 & 214,319 & 0.42 & 1,921 & 287,594 & 0.57 & -0.14 & -34.2 \\
\hline 333 Sparsely vegetated areas & 3,260 & 922,449 & 1.82 & 4,547 & 744,034 & 1.47 & 0.35 & 19.3 \\
\hline 334 Burnt areas & 245 & 55,018 & 0.11 & 259 & 62,557 & 0.12 & -0.01 & -13.7 \\
\hline 335 Glaciers and perpetual snow & 15 & 310 & 0.00 & 20 & 225 & 0.00 & 0.00 & 27.5 \\
\hline 411 Inland marshes & 154 & 54,389 & 0.11 & 109 & 43,780 & 0.09 & 0.02 & 19.5 \\
\hline 412 Peat bogs & 11 & 571 & 0.00 & 18 & 736 & 0.00 & 0.00 & -28.9 \\
\hline 421 Salt marshes & 150 & 29,471 & 0.06 & 315 & 35,641 & 0.07 & -0.01 & -20.9 \\
\hline 422 Salines & 64 & 19,757 & 0.04 & 60 & 18,418 & 0.04 & 0.00 & 6.8 \\
\hline 423 Intertidal flats & 49 & 6,895 & 0.01 & 1,036 & 794 & 0.00 & 0.01 & 88.5 \\
\hline
\end{tabular}


511 Water courses

512 Water bodies

521 Coastal lagoons

522 Estuaries

523 Sea and ocean

\begin{tabular}{rrrrrrrr}
326 & 46,347 & 0.09 & 636 & 48,217 & 0.10 & 0.00 & -4.0 \\
815 & 248,311 & 0.49 & 816 & 238,121 & 0.47 & 0.02 & 4.1 \\
27 & 21,024 & 0.04 & 25 & 8,378 & 0.02 & 0.02 & 60.2 \\
49 & 12,501 & 0.02 & 425 & 11,148 & 0.02 & 0.00 & 10.8 \\
& 0 & 0.00 & & 3,517 & 0.01 & -0.01 & \\
\hline 1,364 & $50,673,293$ & 100.00 & 236,340 & $50,659,463$ & 100.00 & $\mathbf{2 6 . 0 0 *}$ & \\
\hline
\end{tabular}
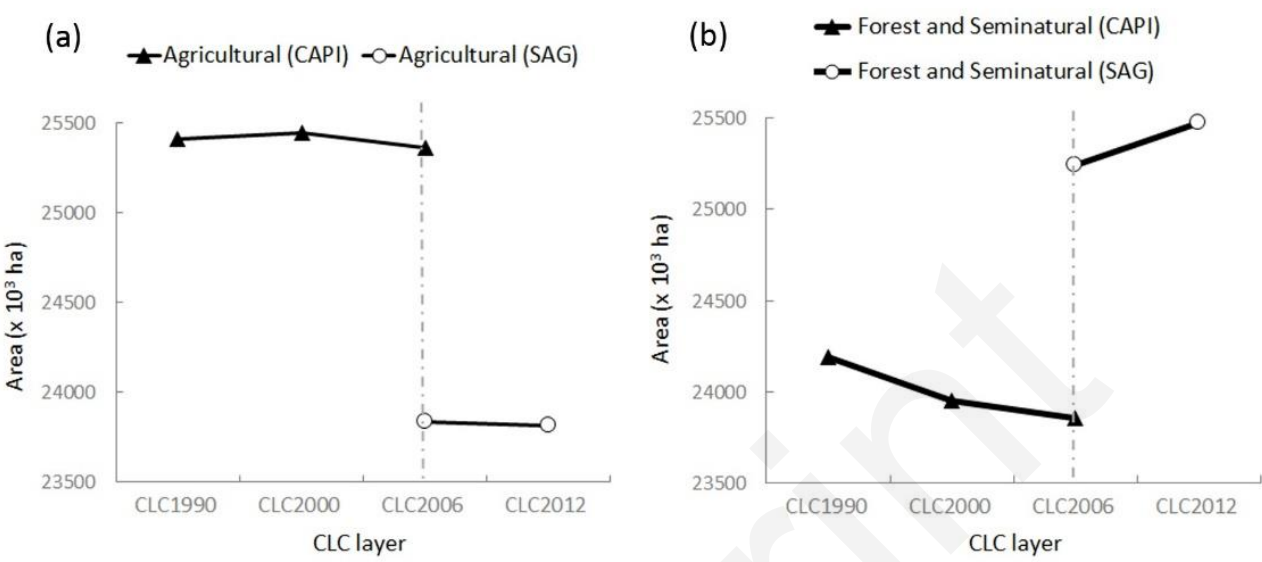

(c)

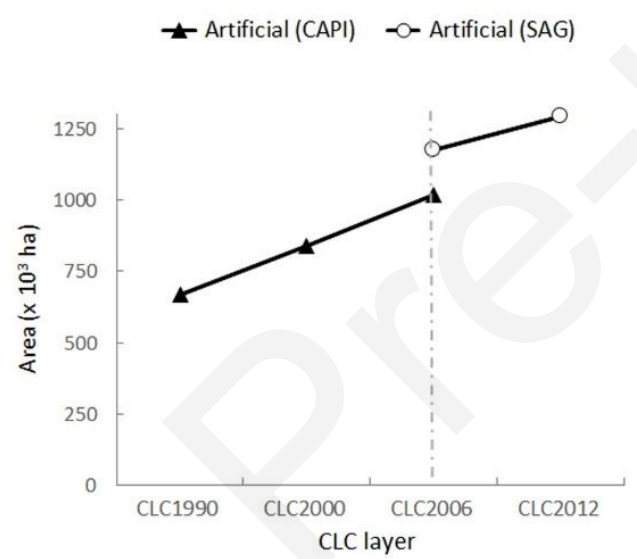

(d)

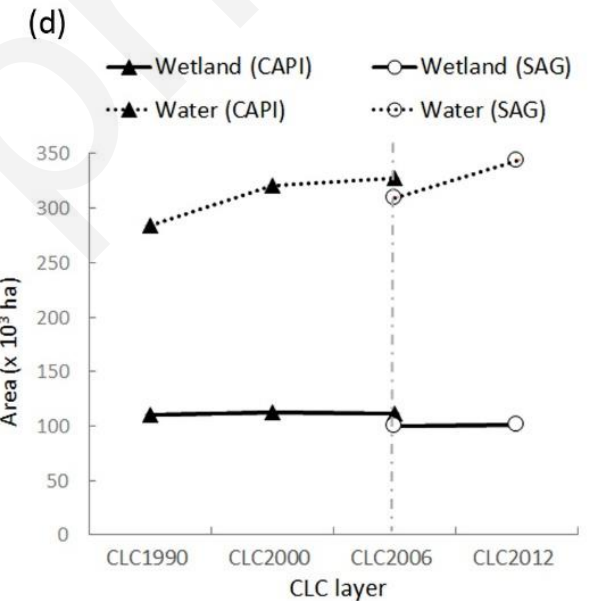

Figure 1. Trends in CLC level 1 classes in Spain for CLC1990-CLC2012 (period 1987-2012). (a) Agricultural, (b) Forest and seminatural, (c) Artificial, (d) Wetland and Water. Outcomes employing CAPI methods (CLC2006initial and previous) are represented with black markers and outcomes employing SAG methods (CLC2006revised and later) with white markers. The change in production methodology occurred in 2006 is indicated with a vertical intermittent line. Source: CLC1990 and CLC2000 values were retrieved from OSE 2011 (Biodiversidad en España). CLC2006 and CLC2012 values were calculated using GIS software. 
Table 3. Commission $(C)$ and omission $(O)$ errors $(\%)$ of seven land cover classes where the disagreement between CLC2006initial and CLC2006revised was highest (see Table 2 for identification of classes with Difference \% values over $+/-1.00 \%)$. The agreement $(\%)$ is italicized. Rows refer to CLC2006revised for $C$ and CLC2006initial for $O$. Depending if the class was under or overestimated (see Table 2 ) the most useful error for evaluation (see section 2.1) is marked with * (i.e., commission or omission error marked as $C^{*}$ or $O^{*}$, respectively). Values over $5.00 \%$ are marked in bold. Summing the error of the 44 classes (Total Error) and the agreement should equal $100.00 \%$.

\begin{tabular}{|c|c|c|c|c|c|c|c|c|c|c|c|c|c|c|c|}
\hline \multirow{2}{*}{\multicolumn{2}{|c|}{ Land cover class }} & \multirow{2}{*}{\multicolumn{2}{|c|}{$\begin{array}{c}\mathbf{2 4 2} \\
\text { Overestimated }\end{array}$}} & \multirow{2}{*}{\multicolumn{2}{|c|}{$\begin{array}{c}\mathbf{2 4 3} \\
\text { Overestimated }\end{array}$}} & \multirow{2}{*}{\multicolumn{2}{|c|}{$\frac{\mathbf{3 1 1}}{\text { Underestimated }}$}} & \multirow{2}{*}{\multicolumn{2}{|c|}{$\begin{array}{c}\mathbf{3 1 2} \\
\text { Underestimated }\end{array}$}} & \multirow{2}{*}{\multicolumn{2}{|c|}{$\begin{array}{c}\mathbf{3 2 1} \\
\text { Underestimated }\end{array}$}} & \multirow{2}{*}{\multicolumn{2}{|c|}{$\begin{array}{c}\mathbf{3 2 2} \\
\text { Underestimated }\end{array}$}} & \multirow{2}{*}{\multicolumn{2}{|c|}{$\begin{array}{c}\mathbf{3 2 4} \\
\text { Overestimated }\end{array}$}} \\
\hline & & & & & & & & & & & & & & & \\
\hline Code & Name & $C^{*}$ & $O$ & $C^{*}$ & $O$ & $C$ & $O^{*}$ & $C$ & $O^{*}$ & $C$ & $O^{*}$ & $C$ & $O^{*}$ & $C^{*}$ & $O$ \\
\hline 111 & Continuous urban fabric & 0.3 & 0.2 & 0.1 & 0.2 & 0.0 & 0.0 & 0.0 & 0.0 & 0.0 & 0.0 & 0.0 & 0.0 & 0.0 & 0.0 \\
\hline 112 & Discontinuous urban fabric & 1.1 & 0.9 & 0.5 & 0.7 & 0.1 & 0.1 & 0.1 & 0.3 & 0.2 & 0.1 & 0.1 & 0.0 & 0.1 & 0.1 \\
\hline 121 & Industrial or commercial units & 0.3 & 0.1 & 0.1 & 0.1 & 0.0 & 0.0 & 0.0 & 0.0 & 0.1 & 0.0 & 0.1 & 0.0 & 0.0 & 0.0 \\
\hline 122 & Road and rail networks & 0.0 & 0.0 & 0.0 & 0.0 & 0.0 & 0.0 & 0.0 & 0.0 & 0.0 & 0.0 & 0.0 & 0.0 & 0.0 & 0.0 \\
\hline 123 & Port areas & 0.0 & 0.0 & 0.0 & 0.0 & 0.0 & 0.0 & 0.0 & 0.0 & 0.0 & 0.0 & 0.0 & 0.0 & 0.0 & 0.0 \\
\hline 124 & Airports & 0.0 & 0.0 & 0.0 & 0.0 & 0.0 & 0.0 & 0.0 & 0.0 & 0.0 & 0.0 & 0.0 & 0.0 & 0.0 & 0.0 \\
\hline 131 & Mineral extraction sites & 0.1 & 0.0 & 0.1 & 0.0 & 0.0 & 0.0 & 0.0 & 0.0 & 0.1 & 0.1 & 0.1 & 0.1 & 0.0 & 0.1 \\
\hline 132 & Dump sites & 0.0 & 0.0 & 0.0 & 0.0 & 0.0 & 0.0 & 0.0 & 0.0 & 0.0 & 0.0 & 0.0 & 0.0 & 0.0 & 0.0 \\
\hline 133 & Construction sites & 0.1 & 0.1 & 0.0 & 0.0 & 0.0 & 0.0 & 0.0 & 0.0 & 0.1 & 0.0 & 0.0 & 0.0 & 0.0 & 0.0 \\
\hline 141 & Green urban areas & 0.0 & 0.0 & 0.0 & 0.0 & 0.0 & 0.0 & 0.0 & 0.0 & 0.0 & 0.0 & 0.0 & 0.0 & 0.0 & 0.0 \\
\hline 142 & Sport and leisure facilities & 0.0 & 0.0 & 0.0 & 0.0 & 0.0 & 0.0 & 0.0 & 0.0 & 0.0 & 0.0 & 0.0 & 0.0 & 0.0 & 0.0 \\
\hline 211 & Non-irrigated arable land & 19.3 & 8.7 & 20.4 & 21.6 & 1.2 & 1.7 & 0.8 & 1.8 & 8.8 & 7.2 & 0.5 & 0.8 & 1.4 & 2.6 \\
\hline 212 & Permanently irrigated land & 6.2 & 2.9 & 1.2 & 2.4 & 0.3 & 0.6 & 0.1 & 0.3 & 0.5 & 0.6 & 0.0 & 0.1 & 0.1 & 0.5 \\
\hline 213 & Rice fields & 0.0 & 0.0 & 0.0 & 0.0 & 0.0 & 0.0 & 0.0 & 0.0 & 0.0 & 0.0 & 0.0 & 0.0 & 0.0 & 0.0 \\
\hline 221 & Vineyards & 8.9 & 2.7 & 1.0 & 1.6 & 0.0 & 0.1 & 0.1 & 0.2 & 0.1 & 0.4 & 0.0 & 0.1 & 0.1 & 0.2 \\
\hline 222 & Fruit trees and berry plantations & 7.6 & 4.9 & 2.5 & 1.9 & 0.1 & 0.1 & 0.2 & 0.4 & 0.1 & 0.6 & 0.0 & 0.0 & 0.3 & 0.4 \\
\hline 223 & Olive groves & 7.7 & 2.5 & 2.8 & 1.4 & 0.2 & 0.2 & 0.2 & 0.3 & 0.3 & 0.5 & 0.0 & 0.0 & 0.5 & 0.5 \\
\hline 231 & Pastures & 2.1 & 1.3 & 2.6 & 3.0 & 0.9 & 1.2 & 0.2 & 0.5 & 4.9 & 1.3 & 1.9 & 1.6 & 0.7 & 0.5 \\
\hline 241 & Annual and permanent crops & 0.2 & 2.2 & 0.1 & 0.6 & 0.0 & 0.0 & 0.0 & 0.1 & 0.0 & 0.0 & 0.0 & 0.0 & 0.0 & 0.0 \\
\hline 242 & Complex cultivation patterns & 25.5 & 53.9 & 7.7 & 16.3 & 0.5 & 2.3 & 0.3 & 1.7 & 0.6 & 3.2 & 1.0 & 2.0 & 0.7 & 1.8 \\
\hline 243 & Agriculture with vegetation & 6.0 & 10.5 & 15.4 & 26.8 & 0.9 & 4.0 & 0.6 & 2.8 & 1.9 & 5.3 & 1.9 & 5.0 & 1.2 & 4.4 \\
\hline 244 & Agro-forestry areas & 0.4 & 0.4 & 1.2 & 2.8 & 5.1 & 4.1 & 0.4 & 0.1 & 5.6 & 5.6 & 0.0 & 0.1 & 4.6 & 3.7 \\
\hline 311 & Broad-leaved forest & 3.1 & 1.0 & 8.1 & 2.3 & 65.3 & 47.8 & 5.3 & 3.0 & 4.0 & 2.4 & 12.5 & 7.2 & 16.0 & 10.5 \\
\hline 312 & Coniferous forest & 2.1 & 0.5 & 5.1 & 1.5 & 3.7 & 4.0 & 67.6 & 56.2 & 1.7 & 2.2 & 4.2 & 3.0 & 15.6 & 7.3 \\
\hline 313 & Mixed forest & 0.7 & 2.1 & 1.8 & 0.7 & 4.0 & 9.0 & 10.8 & 8.5 & 0.8 & 0.8 & 1.5 & 7.2 & 6.0 & 2.6 \\
\hline
\end{tabular}




\begin{tabular}{|c|c|c|c|c|c|c|c|c|c|c|c|c|c|c|c|}
\hline 321 & Natural grasslands & 3.2 & 0.9 & 8.4 & 3.5 & 2.6 & 2.1 & 2.3 & 1.0 & 41.9 & 28.0 & 9.3 & 15.8 & 8.1 & 4.3 \\
\hline 322 & Moors and heathland & 1.0 & 0.5 & 3.9 & 1.2 & 3.8 & 2.3 & 1.5 & 0.8 & 11.8 & 2.2 & 58.2 & 27.4 & 7.2 & 1.9 \\
\hline 323 & Sclerophyllous vegetation & 2.8 & 1.3 & 12.3 & 5.6 & 5.1 & 5.2 & 5.1 & 5.7 & 10.2 & 24.0 & 2.1 & 8.3 & 18.9 & 17.7 \\
\hline 324 & Transitional woodland-shrub & 0.9 & 1.9 & 3.4 & 4.1 & 5.5 & 14.6 & 3.7 & 15.6 & 3.2 & 9.6 & 3.9 & $\mathbf{1 7 . 0}$ & 16.6 & 39.2 \\
\hline 331 & Beaches, dunes, sands & 0.0 & 0.0 & 0.1 & 0.1 & 0.0 & 0.0 & 0.0 & 0.0 & 0.0 & 0.0 & 0.0 & 0.0 & 0.0 & 0.0 \\
\hline 332 & Bare rocks & 0.0 & 0.0 & 0.1 & 0.0 & 0.1 & 0.1 & 0.2 & 0.1 & 0.9 & 0.6 & 0.5 & 0.7 & 0.3 & 0.2 \\
\hline 333 & Sparsely vegetated areas & 0.2 & 0.5 & 0.7 & 1.3 & 0.3 & 0.2 & 0.3 & 0.5 & 1.6 & 4.8 & 1.7 & 3.2 & 0.9 & 1.1 \\
\hline 334 & Burnt areas & 0.0 & 0.0 & 0.0 & 0.0 & 0.0 & 0.0 & 0.0 & 0.1 & 0.1 & 0.1 & 0.2 & 0.1 & 0.2 & 0.2 \\
\hline 335 & Glaciers and perpetual snow & 0.0 & 0.0 & 0.0 & 0.0 & 0.0 & 0.0 & 0.0 & 0.0 & 0.0 & 0.0 & 0.0 & 0.0 & 0.0 & 0.0 \\
\hline 411 & Inland marshes & 0.0 & 0.0 & 0.0 & 0.1 & 0.0 & 0.0 & 0.0 & 0.0 & 0.0 & 0.1 & 0.0 & 0.0 & 0.0 & 0.0 \\
\hline 412 & Peat bogs & 0.0 & 0.0 & 0.0 & 0.0 & 0.0 & 0.0 & 0.0 & 0.0 & 0.0 & 0.0 & 0.0 & 0.0 & 0.0 & 0.0 \\
\hline 421 & Salt marshes & 0.0 & 0.0 & 0.0 & 0.0 & 0.0 & 0.0 & 0.0 & 0.0 & 0.1 & 0.0 & 0.0 & 0.0 & 0.0 & 0.0 \\
\hline 422 & Salines & 0.0 & 0.0 & 0.0 & 0.0 & 0.0 & 0.0 & 0.0 & 0.0 & 0.0 & 0.0 & 0.0 & 0.0 & 0.0 & 0.0 \\
\hline 423 & Intertidal flats & 0.0 & 0.0 & 0.0 & 0.0 & 0.0 & 0.0 & 0.0 & 0.0 & 0.0 & 0.0 & 0.0 & 0.0 & 0.0 & 0.0 \\
\hline 511 & Water courses & 0.0 & 0.0 & 0.0 & 0.0 & 0.0 & 0.1 & 0.0 & 0.0 & 0.0 & 0.0 & 0.0 & 0.0 & 0.0 & 0.1 \\
\hline 512 & Water bodies & 0.0 & 0.0 & 0.1 & 0.1 & 0.1 & 0.1 & 0.0 & 0.0 & 0.2 & 0.1 & 0.0 & 0.1 & 0.1 & 0.1 \\
\hline 521 & Coastal lagoons & 0.0 & 0.0 & 0.0 & 0.0 & 0.0 & 0.0 & 0.0 & 0.0 & 0.0 & 0.0 & 0.0 & 0.0 & 0.0 & 0.0 \\
\hline 522 & Estuaries & 0.0 & 0.0 & 0.0 & 0.0 & 0.0 & 0.0 & 0.0 & 0.0 & 0.0 & 0.0 & 0.0 & 0.0 & 0.0 & 0.0 \\
\hline \multirow[t]{2}{*}{523} & Sea and ocean & 0.0 & 0.0 & 0.0 & 0.0 & 0.0 & 0.0 & 0.0 & 0.0 & 0.0 & 0.0 & 0.0 & 0.0 & 0.0 & 0.0 \\
\hline & Total Error (\%) & $74.5^{*}$ & 46.1 & $84.6^{*}$ & 73.2 & 34.7 & $52.2 *$ & 32.4 & $43.8 *$ & 58.1 & $72.0^{*}$ & 41.8 & $72.6^{*}$ & 83.4 & $60.8 *$ \\
\hline
\end{tabular}




\subsection{Differences in European Countries}

Among nine countries compared (Table 4) a group of five including Spain, Germany, Austria, Ireland, and the UK showed high disagreement and differences between CLC2000 and CLC2006initial layers and those produced later (CLC2006revised and CLC2012). There is a marked breakpoint in CLC2006revised highlighted by column Dif'06 values deviating from zero and SDV global values over 1.0\% (indicated with "**" in Table 4). All five countries with marked change in LC trends have modified their CLC production methodology for the last update. SDV values demonstrate different level of severity in the discrepancies due to change in methodology, which seems to be more severe in the UK (12.1\% of difference), followed by Spain (6.2\%) and Germany (4.6\%). The UK reverted from generalization to CAPI whereas Spain and Germany changed from CAPI to SAG (Feranec et al., 2016b). Interestingly Austria and Ireland CLC versions - which methods also changed from CAPI to SAG_-show higher agreement and visual similarity between CLC2006initial and CLC2006revised versions than Spain and Germany. Values for countries that have kept production methodologies unchanged (CAPI in France, Portugal, and the Netherlands, and generalization in Finland) show agreement and continuity without major alteration in trends. Visual examples of disagreement are displayed in Figure 2, demonstrating the impact a change in methods has on LC maps. Perhaps the most remarkable visual impact is noticed on the Spain/Portugal figures, with a remarkable increase in polygon complexity on one side (Spain) whilst LC is apparently stable on the other side (Portugal). The Austria/Germany case demonstrate a similar effect on both sides, whereas Ireland changes are visually less relevant and the UK shows some loss of detail in polygon delineation.

\section{Discussion}

Estimation of long-term LC trends can be affected by methodological modifications in map production, as demonstrated by the comparison of CLC trends in the Spanish case employing CLC2006initial and CLC2006revised. These two versions of the same LC were obtained with CAPI and SAG, respectively. The effects of methodological variations in the Spanish case can only be actually evaluated for 2006, the date of methodological transition, because CLC2006revised and CLC2006initial represent the same LC but characterized by different methodologies. To estimate how the methodological variation has affected LC trends one option is to assume that the same amount of error found in 2006 affects other layers/dates. The pattern of over- and underestimations identified in this comparison can be extended to previous inventories produced with CAPI (i.e., CLC2000 and CLC1990). For instance, the $2.5 \%$ underestimation of grassland in CLC2006initial with respect to CLC2006revised is probably similar when comparing CLC2000 with CLC2006 (or with CLC2012). To obtain figures of real change in this hypothetical case, the 2006 methodological change estimated in this study should be added to the 2000-2006 change from comparison of layers. Understanding these patterns would facilitate and improve the interpretation of long-term comparisons (e.g., Fernández-Nogueira and Corbelle-Rico 2018, for the Iberian Peninsula). Employing graphics similar to those in Figure 1 contribute to better estimate real trends of change. The difference percentages (Difference (\%) and NC (\%)) presented for each LC class in Tables 1 and 2 are also means to detect and correct the "artificial" change and help to 
estimate "real" change, because they are quantitative estimations below and over real figures, that is, under- or over-estimation.

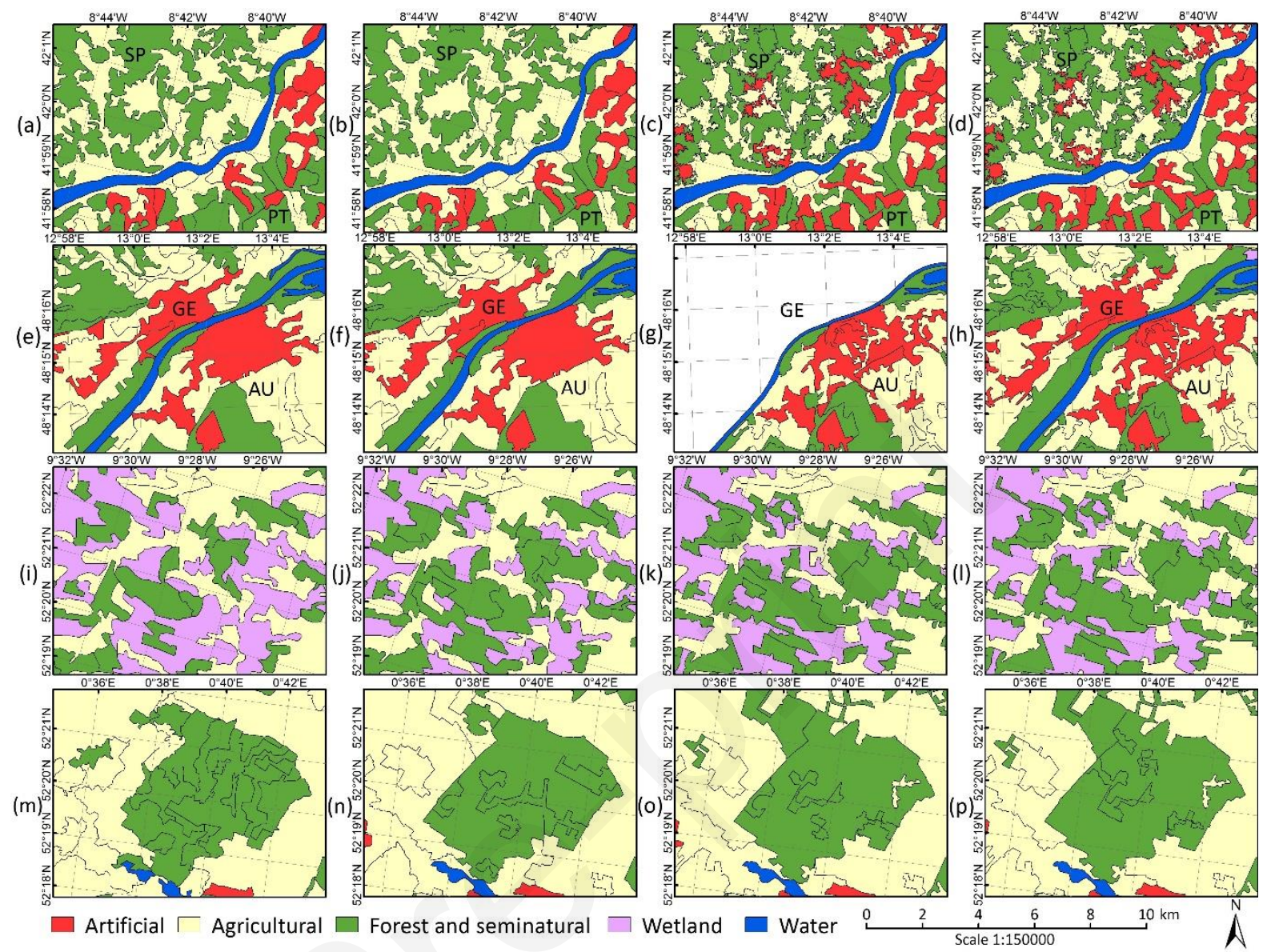

Figure 2. Visual comparison between CLC versions in various European countries: Spain (SP) and Portugal (PT) ((a), (b), (c), (d)), Germany (GE) and Austria (AU) ((e), (f), (g), (h)), Ireland ((i), (j), (k), (l)) and the UK ((m), (n), (o), (p)). CLC level 3 polygons are outlined and CLC level 1 polygons are code-coloured. First column ((a), (e), (i), (m)) represents CLC2000revised, second column ((b), (f), (j), (n)) CLC2006initial; third column ((c), (g), (k), (o)) CLC2006revised and fourth column ((d), (h), (l), (p)) CLC2012initial. Sources: European CLC seamless data coverage version 18.5 for CLC2000revised, CLC2006revised and CLC2012initial; CLC version 17 for CLC2006initial. For Germany CLC2006revised is not available for download from the EEA website because a non-standard methodology was applied. In Germany CLC2006 version 18.5 is the same as version 17 and corresponds to CLC2006initial. 
Table 4. CLC level 1 class percentages along CLC2000 (00), CLC2006initial (06 ${ }_{\mathrm{i}}$ ), CLC2006revised (06 ${ }_{\mathrm{r}}$ ) and CLC2012 (12) in nine European countries and the difference between CLC2006initial and CLC2006revised (06 i-r $_{\text {) }}$. Source: EEA, 2011; EEA, 2017. The production methodology (CAPI or SAG) is indicated. SDV (see Equation 1) is indicated with *. Differences over 1.00\% are in bold.

\begin{tabular}{|c|c|c|c|c|c|}
\hline \multirow{3}{*}{ Land cover class } & \multicolumn{5}{|c|}{ Spain } \\
\hline & 00 & $06_{i}$ & $06 \mathrm{r}$ & 12 & \multirow{2}{*}{$06_{\text {i-r }}$} \\
\hline & CAPI & CAPI & SAG & SAG & \\
\hline Artificial & 1.8 & 2.0 & 2.5 & 2.7 & 0.42 \\
\hline Agricultural & 50.1 & 50.1 & 47.1 & 46.9 & -3.01 \\
\hline Forest and seminatural & 47.2 & 47.0 & 49.7 & 49.6 & 2.66 \\
\hline Wetlands & 0.2 & 0.2 & 0.2 & 0.2 & -0.02 \\
\hline \multirow[t]{3}{*}{ Water bodies } & 0.6 & 0.7 & 0.6 & 0.6 & -0.05 \\
\hline & 100.0 & 100.0 & 100.0 & 100.0 & *6.16 \\
\hline & \multicolumn{5}{|c|}{ Ireland } \\
\hline \multirow{2}{*}{ Land cover class } & 00 & $06_{i}$ & $06_{r}$ & 12 & \multirow{2}{*}{$06_{\mathrm{i}-\mathrm{r}}$} \\
\hline & CAPI & CAPI & SAG & SAG & \\
\hline Artificial & 2.0 & 2.3 & 2.5 & 2.5 & 0.16 \\
\hline Agricultural & 67.0 & 66.4 & 67.2 & 67.0 & 0.83 \\
\hline Forest and seminatural & 12.1 & 12.8 & 12.7 & 13.0 & -0.07 \\
\hline Wetlands & 16.7 & 16.2 & 15.5 & 15.4 & -0.76 \\
\hline \multirow[t]{3}{*}{ Water bodies } & 2.2 & 2.2 & 2.1 & 2.1 & -0.16 \\
\hline & 100.0 & 100.0 & 100.0 & 100.0 & $* 1.98$ \\
\hline & \multicolumn{5}{|c|}{ France } \\
\hline \multirow{2}{*}{ Land cover class } & 00 & $06_{i}$ & $06_{r}$ & 12 & \multirow{2}{*}{$06_{\mathrm{i}-\mathrm{r}}$} \\
\hline & CAPI & CAPI & SAG & SAG & \\
\hline Artificial & 5.0 & 5.1 & 5.4 & 5.5 & 0.27 \\
\hline Agricultural & 59.6 & 59.5 & 59.2 & 59.0 & -0.33 \\
\hline Forest and seminatural & 33.9 & 33.9 & 33.9 & 33.9 & 0.01 \\
\hline Wetlands & 0.7 & 0.7 & 0.7 & 0.7 & 0.00 \\
\hline Water bodies & 0.8 & 0.8 & 0.9 & 0.9 & 0.05 \\
\hline
\end{tabular}

\begin{tabular}{|c|c|c|c|c|}
\hline \multicolumn{5}{|c|}{ Germany } \\
\hline 00 & $06_{i}$ & $06 r$ & 12 & \multirow{2}{*}{$06_{\text {i-r }}$} \\
\hline CAPI & CAPI & SAG & SAG & \\
\hline 8.2 & 8.3 & 9.3 & 9.4 & 0.96 \\
\hline 58.9 & 58.8 & 56.5 & 56.4 & -2.30 \\
\hline 30.2 & 30.2 & 31.5 & 31.5 & 1.30 \\
\hline 1.2 & 1.2 & 1.2 & 1.2 & 0.00 \\
\hline 1.4 & 1.5 & 1.5 & 1.5 & 0.04 \\
\hline 100.0 & 100.0 & 100.0 & 100.0 & $* 4.60$ \\
\hline
\end{tabular}

\begin{tabular}{rrrrr}
\multicolumn{5}{c}{ Austria } \\
\cline { 2 - 4 } 00 & \multicolumn{1}{c}{ 06 $_{\text {i }}$} & \multicolumn{1}{c}{ 06r } & \multicolumn{1}{c}{$\mathbf{1 2}$} & \multirow{2}{*}{ 06 $_{\text {i-r }}$} \\
\cline { 1 - 4 } CAPI & CAPI & \multicolumn{1}{c}{ SAG } & \multicolumn{1}{c}{ SAG } & \\
\hline 4.8 & 4.9 & 5.5 & 5.6 & 0.65 \\
32.5 & 32.4 & 32.2 & 32.1 & -0.24 \\
61.7 & 61.6 & 61.2 & 61.1 & -0.46 \\
0.3 & 0.3 & 0.3 & 0.3 & -0.01 \\
0.8 & 0.8 & 0.9 & 0.9 & 0.05 \\
\hline 100.0 & 100.0 & 100.0 & 100.0 & $* \mathbf{1 . 4 2}$
\end{tabular}

\begin{tabular}{rrrrc}
\hline \multicolumn{1}{c}{ 00 } & \multicolumn{1}{c}{ 06 } & 06r & \multicolumn{1}{c}{ 12 } & \multirow{2}{*}{ 06 $_{\text {i-r }}$} \\
\cline { 1 - 4 } CAPI & CAPI & SAG & \multicolumn{1}{c}{ SAG } & \\
\cline { 1 - 3 } 7.7 & 7.8 & 8.2 & 8.3 & 0.42 \\
57.6 & 57.5 & 55.7 & 55.6 & $\mathbf{- 1 . 7 7}$ \\
28.4 & 28.5 & 24.2 & 24.1 & $\mathbf{- 4 . 3 0}$ \\
5.3 & 5.3 & 10.6 & 10.6 & $\mathbf{5 . 2 9}$ \\
1.0 & 1.0 & 1.4 & 1.4 & 0.36 \\
\hline 100.0 & 100.0 & 100.0 & 100.0 & $* \mathbf{1 2 . 1 3}$
\end{tabular}

\begin{tabular}{|c|c|c|c|c|}
\hline \multicolumn{5}{|c|}{ Finland } \\
\hline 00 & $06_{i}$ & $06_{r}$ & 12 & \multirow{2}{*}{$06_{\text {i-r }}$} \\
\hline CAPI & CAPI & SAG & SAG & \\
\hline 1.4 & 1.4 & 1.3 & 1.4 & -0.09 \\
\hline 8.6 & 8.8 & 8.6 & 8.6 & -0.21 \\
\hline 74.0 & 73.8 & 74.2 & 74.2 & 0.37 \\
\hline 6.7 & 6.6 & 6.4 & 6.4 & -0.20 \\
\hline 9.3 & 9.3 & 9.5 & 9.5 & 0.13 \\
\hline 100.0 & 100.0 & 100.0 & 100.0 & *1.00 \\
\hline
\end{tabular}

\begin{tabular}{rrrrc}
\multicolumn{5}{c}{ Portugal } \\
\cline { 2 - 4 } 00 & \multicolumn{1}{c}{ 06 } & \multicolumn{1}{c}{ 06r } & \multicolumn{1}{c}{ 12 } & \multirow{2}{*}{ 06 $_{\text {i-r }}$} \\
\cline { 1 - 4 } CAPI & CAPI & \multicolumn{1}{c}{ SAG } & \multicolumn{1}{c}{ SAG } & \\
\cline { 1 - 4 } 3.2 & 3.5 & 3.9 & 4.0 & 0.34 \\
47.5 & 46.9 & 46.5 & 46.4 & -0.47 \\
47.8 & 47.8 & 47.8 & 47.8 & 0.04 \\
0.3 & 0.3 & 0.4 & 0.4 & 0.06 \\
1.2 & 1.4 & 1.5 & 1.5 & 0.04 \\
\hline 100.0 & 100.0 & 100.0 & 100.0 & $* 0.94$
\end{tabular}

\begin{tabular}{rrrrc}
\multicolumn{5}{c}{ Netherlands } \\
\cline { 1 - 4 } 00 & 06 $_{\text {i }}$ & 06r & 12 & \multirow{2}{*}{ 06 $_{\text {i-r }}$} \\
\cline { 1 - 4 } CAPI & CAPI & SAG & SAG & \\
\hline 11.9 & 12.8 & 12.9 & 13.4 & 0.10 \\
62.5 & 61.4 & 61.2 & 60.4 & -0.20 \\
10.2 & 10.3 & 10.4 & 10.6 & 0.11 \\
7.0 & 7.0 & 7.0 & 7.0 & -0.06 \\
8.4 & 8.5 & 8.5 & 8.5 & 0.05 \\
\hline 100.0 & 100.0 & 100.0 & 100.0 & $* 0.53$
\end{tabular}


The change in production methodology in CLC2006revised and following CLC layers (CLC2012, CLC2018) provides higher accuracy in the delimitation of polygons, particularly of LC classes that seem to be underestimated in 2006 and before, that is, discontinuous urban fabric (112), industrial or commercial units (121), salt marshes (421), water courses (511), crops, and forests. These LC classes had previously been classified in either spectrally similar classes, thematically more generic, or in heterogeneous mosaics. It is in compound classes like complex cultivation patterns and agriculture with areas of natural vegetation where the change in methodology has had a more important impact. These classes, highly dependent on the scale effect and the degree of detail in the resolution of digitization, were overestimated in the original version. Another comparison performed at the local scale in Asturias (Spain) by García-Álvarez and Camacho (2017) had similar conclusions, pointing out that mixed classes had the lowest agreement between CLC2006initial and CLC2006revised, in contrast to homogenous and relatively non-fragmented classes.

More than one million ha were classified in CLC2006initial as agriculture with areas of natural vegetation (243) (Table 2), and in CLC2006revised as non-irrigated arable land $(20.4 \%)$, some type of shrub-land $(19.6 \%)$, natural grasslands $(8.4 \%)$ and forests $(15.0 \%)$ (Table 3). Overall, transitional woodland-shrub (324) was the LC class with larger differences between CLC2006initial and CLC2006revised (Table 2), due to misclassification into forest, shrub-land, and natural grasslands (Table 3). These types of error may in part be due to the definition of the LC class itself, its transitional character, and also to difficulties for identification and digitization onscreen at 1:100000 scale. Transitional woodland-shrub differs from forest and sclerophyllous vegetation in the canopy height and canopy cover fraction of isolated trees and shrubs (OSE 2006). Detailed discrimination of these classes requires field observations and very detailed ancillary data. This is possibly the most confusing CORINE LC class as indicated by previous validation studies in Spain (Barreira et al., 2012) and Portugal (Caetano et al., 2006).

For analysis of LC change dynamics, enquiring CLCchange databases is encouraged (CNIG Spain and EEA), and direct comparison of individual CLC layers is discouraged (Büttner 2016). CLCchange layers enable identification of real LC changes discerning them from artificial changes due to methodological differences. Nevertheless, when comparing individual or status CLC layers is necessary (e.g., for assessment of change rates and dynamics, García-Álvarez et al., 2017) authors should be aware of the issues raised here, and when comparing CLC2006revised with CLC2012 they should be prepared to obtain considerable differences to other studies employing CLC2006initial (OSE, 2011; MartínezFernández et al., 2015; Bonet et al., 2016).

CLCchange is not a wall-to-wall layer and it is not affected by changes in methodological production of the status layers, because it is always based on the revised version of the "before" reference year, which is derived employing the same methodology as the "after" status layer. CLCchange is a vector map of change polygons that has two attributes: CLC code for year 1 and CLC code for year 2 (e.g., 211-321). For analysis of LC class total areas at different dates, the CLCchange layer is not practical: total gains and losses for each LC class should be calculated and added to the initial status layer. This process should be repeated for each CLC period (i.e., 1990-2000, 2000-2006, 2006-2012). In addition, this option combining information from a status and a change layer requires consideration of the different MMU (25.0 ha and 5.0 ha, respectively). Moreover, GarcíaÁlvarez et al. (2017) stated some reasons why CLCchange is not suitable for all user needs, for instance when the rates and pattern of change are needed, to generate derived products 
updated on the basis of the new maps, or in studies determining drivers of change or territorial dynamics - which require the status layer as input.

In spite of the higher detail of the new CLC maps, García-Álvarez and Camacho (2017) identified important uncertainty elements, especially the fact that its complexity is not of the level expected at a 1:100000 scale -it has more complexity than anticipated. This complexity refers to the geometry, perimeter shape, patch fragmentation, and the presence of corridors that violate the minimum width rule $(100 \mathrm{~m})$. In contrast to the Spanish case, Austria and Ireland (Figure 2, Table 4), which have also employed SAG methods to produce the most recent CLC layers, have a higher agreement and visual similarity with the previous CLC versions. Implementing geometrical simplification mechanisms to adapt the resulting geometry to the perimeters of the previous patches (e.g., in Ireland-Lydon and Smith, 2014), or performing geometric and semantic transformation as an iterative process in four steps of MMU upscaling (e.g., in Austria-Banko et al., 2014) seem to be effective mechanisms. In the case of Norway, the generalization process is explained with detail in Heggem and Strand (2010). It is basically a process simulating manual delineation, followed by refinement methods adapted to some specific classes like heterogeneous agriculture or green urban area. Similarly, in Finland the generalization is first implemented for each main level 1 class separately, as well as for some rare classes (Härmä et al., 2005). Some of these countries reporting their generalization methods indicate the necessity to perform visual and manual steps like edition, checking or control, to achieve acceptable results. The CLC scheme characteristics make the automation of generalization based on statistical or quantitative criteria unsatisfactory for map production, and some expert intervention (e.g., with PI, Feranec et al., 2007) becomes necessary to produce quality maps avoiding inconsistencies and uncertainties (García-Álvarez and Camacho, 2017). Improving the process of generalization may be beneficial in the Spanish case, by introducing expert criteria and improving the smoothing and simplification of the polygon boundaries to make them spatially and geometrically more coincident and adapted to the previous CLC layers. Reporting specifically and with detail about the generalization methods, rules, and techniques applied is also necessary for the research community.

Generalization methods and the characteristics of the source maps lead to differences in each country (Gallego et al., 2000). Likewise, the quality of the generalization and the level of agreement with previous CLC versions vary depending on the complexity, fragmentation, and heterogeneity of the landscape throughout the country (García-Álvarez and Camacho, 2017). In the case of Spain, heterogeneity and fragmentation are higher in the north, some Mediterranean areas, and in mountain areas, and lower in landscapes like the central Castilian plain (Molinero, 2014; García-Álvarez and Camacho, 2017), making regional and local LC change assessments very specific.

The methodological change in CLC production, from photointerpretation to generalization has brought important differences that should be accounted for. Some countries chose to keep temporal consistency in CLC versions, maintaining temporal continuity of CAPI methods (France, Portugal and The Netherlands) or generalization methods (Finland). A change in methodology followed by countries like Spain is justified by the achievement of consistency among national databases and maps, as well as considerable reductions in production time and cost (Hazeu et al., 2016), and incorporation of technical improvements (Smith, 2014). In the case of the Netherlands, its small size makes it not economically cost effective to develop a bottom-up approach fully integrating national LULC and the Dutch CLC (Hazeu et al., 2016). 


\section{Conclusions}

Methodological changes in production of the most recent CLC maps in Spain (CLC2012 and CLC2006revised) have had consequences for analysis and interpretation of LC trends over the long-term series (CLC1990-CLC2012), which in Spain corresponds to period 1987-2011 according to the reference images. Substituting computer-assisted photointerpretation with SAG of a more detailed map involved more accurate accounts of LC classes such as urban fabric and heterogeneous agricultural areas. In long-term LC comparative works, change due to map production - in addition to real change due to disturbance or natural developmentshould be carefully considered and reported. Retrospective interpretation of trends allows identifying LC classes which had been underestimated (artificial areas, crops, forest, and natural grasslands) and overestimated (heterogeneous agricultural and transitional woodlandshrub) in previous CLC versions. The consistency of the LC map series in Spain would benefit from detailed reporting of the map generalization methods.

\section{Acknowledgments}

This work was funded by the Spanish Ministry of Science, Innovation and University through project AGL2016-76769-C2-1-R "Influence of natural disturbance regimes and management on forests dynamics, structure and carbon balance (FORESTCHANGE)". PRB was supported by TALENTO Fellow Programme (Comunidad de Madrid, 2016-T2/AMB-1665, Spain). Four reviewers are thanked for their positive comments and suggestions for improvement of the manuscript.

\section{Disclosure statement}

No potential conflict of interest was reported by the authors

\section{References}

Banko, G., R. Mansberger, H. Gallaun, R. Grillmayer, R. Prüller, M. Riedl, W. Stemberger, K. Steinnocher, and A. Walli. 2014. "Land Information System Austria (LISA)." In: Manakos, I., Braun, M. (Eds.), Land Use and Land Cover Mapping in Europe. Practices and Trends. Springer, Dordrecht Heidelberg New York London, pp. 237-254. DOI: 10.1007/97894-007-7969-3

Barreira, P., V. González, and J. Bosque. 2012. "Detección de errores temáticos en el CORINE Land Cover a través del estudio de cambios: Comunidad de Madrid (2000-2006)." Estudios Geográficos 73 (272): 7-34.

Bonet, A., P. Ruiz-Benito, B. Terrones, J. Martínez-Fernández, M.A. Zavala. 2016. “Capítulo 5.4. Diagnóstico: Ocupación del suelo en España” In Bases científico-técnicas para la Estrategia estatal de infraestructura verde y de la conectividad, edited by F. Valladares, P. Gil and A. Forner, 182-203, Madrid: Ministerio de Agricultura y Pesca, Alimentación y Medio Ambiente. Accessed $\quad$ May 2019 https://www.miteco.gob.es/es/biodiversidad/temas/ecosistemas-yconectividad/basescientifico-tecnicaseeivcre tcm30-479558.pdf 
Bossard, M., J. Feranec, J. Otahel. 2000. "CORINE Land Cover Technical Guide Addendum 2000. Technical Report No 40.” European Environment Agency, Copenhagen, Denmark, 105

pp. http://www.dmu.dk/fileadmin/Resources/DMU/Udgivelser/CLC2000/technical guide addenum.pdf (accessed December 2013).

Büttner, G. 2014. "CORINE Land Cover and Land Cover Change Products." In: Manakos, I., Braun, M. (Eds.), Land Use and Land Cover Mapping in Europe: Current Practice, Trends and Future. Springer, Netherlands, pp. 55-74.

Büttner, G. 2016. "Chapter 7. CORINE Land Cover Products." In: European Landscape Dynamics: CORINE land cover data. CRC Press. Feranec, J., Soukup, T., Hazeu, G., and Jaffrain, G. (Eds.). p. 275-304. DOI: 10.1201/9781315372860-27.

Caetano, M., F. Mata, and S. Freire. 2006. "Accuracy assessment of the Portuguese CORINE Land Cover map." In: Global Developments in Environmental Earth Observation from Space (A. Marçal, editor), Millpress, Rotterdam, pp. 459-467.

Chuvieco, E. 2010. “Teledetección ambiental: la observación de la Tierra desde el espacio.” Barcelona, España, Ed. Ariel, 586 pp. ISBN: 9788434434981

CNIG, 2013. “Corine Land Cover España 2006.” Release data: 19-07-2013. Centro Nacional de información Geográfica. Centro de Descargas. Available in: http://centrodedescargas.cnig.es/

Cole, B., S. King, B. Ogutu, D. Palmer, G. Smith, H. Balzter. 2015. "Corine land cover 2012 for the UK, Jersey and Guernsey." NERC Environmental Information Data Centre. https://doi.org/10.5285/32533dd6-7c1b-43e1-b892-e80d61a5e-a1d (accessed in August 2018)

EEA, 2011. "Land cover 2006 and changes country analysis." European Environment Agency Available in: https://www.eea.europa.eu/data-and-maps/figures/land-cover-2006and-changes\#tab-documents

EEA, 2014. "Corine Land Cover 2006 seamless vector data. Version 17.” Release date: 212-2013. European Environment Agency. Available in: http://www.eea.europa.eu/data-andmaps/data/clc-2006-vector-data-version-3

EEA, 2016. "Corine Land Cover (CLC), Version 18.5.1." Release date: 19-09-2016. European Environment Agency. Available in: https://land.copernicus.eu/paneuropean/corine-land-cover

EEA, 2018. “Corine Land Cover (CLC) 2018, Version 20b2.” Release date: 21-12-2018. European Environment Agency. Available in: https://land.copernicus.eu/paneuropean/corine-land-cover/clc2018 
EEA, 2017. "Land cover country fact sheets 2012. Reports by country. European Environment Agency." Available in: https://www.eea.europa.eu/themes/landuse/land-covercountry-fact-sheets

EEA, 2007. "CLC2006 Technical Guidelines. Technical Report No 17." European Environment Agency, Cophenhagen, $66 \quad$ pp. https://www.eea.europa.eu/publications/technical report 200717 (accessed in August 2018)

Feranec, J., G. Hazeu, S. Christensen, G. Jaffrain. 2007. "Corine land cover change detection in Europe (case studies of the Netherlands and Slovakia)." Land Use Policy 24(1): 234-247.

Feranec, J., G. Hazeu, T. Soukup. 2016b. "Chapter 5. Interpretation of Satellite Images. In: European Landscape Dynamics: CORINE land cover data.” CRC Press. Feranec, J., Soukup, T., Hazeu, G., and Jaffrain, G. (Eds.). p. 33-41.

Feranec, J., T. Soukup, G. Hazeu, and G. Jaffrain. (Eds.). 2016a. "European landscape dynamics: CORINE land cover data." Boca Raton: CRC Press. Taylor and Francis Group. 337 p. ISBN: 978-1-4822-4466-3

Fernández-Nogueira, D, and E. Corbelle-Rico. 2018. "Land use changes in Iberian Peninsula." Land 7 (99): doi: 10.3390/land7030099.

Gallego, F. J., P. Escribano, S. Christensen. 2000. "Comparability of landscape diversity indicators in the European Union. From Land cover to landscape diversity in the European Union, 84-97. Report of the European Commission. Available in: http://agrienv.jrc.ec.europa.eu/publications/pdfs/diversityCORINE MARS.pdf

García-Álvarez, D., and M.T. Camacho Olmedo. 2017. "Changes in the methodology used in the production of the Spanish CORINE: Uncertainty analysis of the new maps." International Journal of Applied Earth Observation and Geoinformation 63: 55-67.

Härmä, P., R. Teiniranta, M. Törmä, R. Repo, E. Järvenpää, E. Kallio. 2005. "CLC2000 Finland: Final report." Finnish Environment Institute, Geoinformatics and Land Use Division, vol. 9 (num 2011), $66 \quad$ pp. Available in http://www.syke.fi/download/noname/\%7BB4231601-439E-4903-B6FC2F2FE718B465\% 7D/39571 (accessed August 2018).

Hazeu, G.W., G. Büttner, A. Arozarena, N. Valcarcel., J. Feranec, and G. Smith. 2016. "Chapter 26. Detailed CLC Data: Member States with CLC Level 4/Level 5 and (Semi-) Automated Solutions.” In: European Landscape Dynamics: CORINE land cover data. CRC Press. Feranec, J., Soukup, T., Hazeu, G., and Jaffrain, G. (Eds.). p. 275-304. DOI: 10.1201/9781315372860-27.

Heggem, E.S.F., and G.H. Strand. 2010. "CORINE Land Cover 2000. The Norwegian CLC200 project.” Norsk Institutt for Skog og Landskap. Pb 115, NO-1431, 23 pp. Available online at: https://land.copernicus.eu/eagle/files/eagle-relatedprojects/no clc2000 norwegian-project (accessed August 2018) 
Heymann, Y., C. Steenmans, G. Croissille, and M. Bossard. 1994. "Corine Land Cover- A technical guide." Office for Official Publications of European Communities.CEC, Luxembourg, http://www.eea.europa.eu/publications/COR0-landcover (accessed in August 2018).

Hovenbitzer, M., F. Emig, C. Wende, S. Arnold, M. Bock, and S. Feigenspan. 2014. "Digital land cover model for Germany -DLM-DE." In: Manakos, I., Braun, M. (Eds.), Land Use and Land Cover Mapping in Europe. Practices and Trends. Springer, Dordrecht Heidelberg New York London, pp. 255-272. DOI: 10.1007/978-94-007-7969-3

Lydon, K., and G. Smith. 2014. “Corine Land Cover 2012 Ireland Final Report.” Wexford. Available online at: https://www.epa.ie/pubs/data/corinedata/CLC2012 IE Final Report.pdf (accessed in August 2018)

Maes J., B. Egoh, L.Willemen, C. Liquete, P. Vihervaara, J.P. Schägner, B. Grizzetti, E.G. Drakou, A. La Notte, G. Zulian, F. Bouraoui, M.L. Paracchini, L. Braat, and G. Bidoglio. 2012. "Mapping ecosystem services for policy support and decision making in the European Union." Ecosystem Services 1: 31-39.

Martínez-Fernández, J., P. Ruiz-Benito, M.A. Zavala. 2015. "Recent land cover changes in Spain across biogeographical regions and protection levels: Implications for conservation policies." Land Use Policy 44: 62-75.

Molinero, F. 2014. "Atlas de los paisajes agrarios de España." Ministerio de Agricultura, Alimentación y Medio Ambiente. Centro de Publicaciones. Madrid. 1071 pp. ISBN: 978-84491-1347-5

OSE. 2006. "Cambios en la ocupación del suelo en España. Implicaciones para la sostenibilidad." Observatorio de la Sostenibilidad en España. Ministerio de Medio Ambiente, Madrid, España. Ed. Mundi-Prensa, 485 pp. ISBN: 84-8476-287-4

OSE. 2011. "Biodiversidad en España: Base de la sostenibilidad ante el cambio global." Observatorio de la Sostenibilidad en España. Ministerio de Medio Ambiente, Medio Rural y Marino, Fundación Biodiversidad y Fundación General de la Universidad de Alcalá, Madrid, España, Ed. Mundi-Prensa, 503 pp. ISBN: 978-84-8476-433-5

Pontius, R.G.Jr., E. Shusas, and M. McEachern. 2004. "Detecting important categorical land changes while accounting for persistence." Agriculture, Ecosystems and Environment 101 (2-3): 251-268.

Smith, G.M. 2014. "Land Use-Land Cover Mapping in Europe: Examples from UK. In: Manakos, I., Braun, M. (Eds.), Land Use and Land Cover Mapping in Europe. Practices and Trends." Springer, Dordrecht Heidelberg New York London, pp. 273-282. DOI: 10.1007/978-94-007-7969-3

Soukup, T., J. Feranec, G. Hazeu, G. Jaffrain, M. Jindrova, M. Kopecky, and E. Orlitova. 2016. "Chapter 10. CORINE Land Cover 1990 (CLC1990): Analysis and Assessment. In: 
European Landscape Dynamics: CORINE land cover data.” CRC Press. Feranec, J., Soukup, T., Hazeu, G., and Jaffrain, G. (Eds.). p. 71-78. DOI: 10.1201/9781315372860-27.

Turner B.L., E.F. Lambin, and A. Reenberg. 2007. "The emergence of land change science for global environmental change and sustainability." Proceedings of the National Academy of Sciences 104(52) 20666-20671

Weiers, S., G. Groom, and M. Wissen. 2002. "Comparability and subjectivity of land cover maps produced with digital image classification techniques: some recent experiences from Denmark and northern Germany." Geografisk Tidsskrift-Danish Journal of Geography 102(1): 59-77. 